Supporting Information

\title{
Are Diamond Nanoparticles Cytotoxic?
}

\author{
Amanda M. Schrand ${ }^{1}$, Houjin Huang ${ }^{1}$, Cataleya Carlson ${ }^{2}$, John J. Schlager ${ }^{2}$, Eiji \\ Osawa ${ }^{3}$, Saber M. Hussain ${ }^{2 *}$, and Liming Dai ${ }^{i^{*}}$
}

${ }^{1}$ University of Dayton, Department of Chemical and Materials Engineering, 300 College Park, Dayton, OH 45469-0240, USA

${ }^{2}$ Applied Biotechnology Branch, Human Effectiveness Directorate, Air Force Research Laboratory, Wright-Patterson AFB, OH 45433-5707, USA

${ }^{3}$ NanoCarbon Research Institute, Ltd., Kashiwa, Chiba 277-0882, Japan 
ND Surface Modification and Dispersion. The as-received nanodiamonds (ND-raw) were subjected to the treatment with acid $\left(70 \% \mathrm{HNO}_{3}\right.$ and $98 \% \mathrm{H}_{2} \mathrm{SO}_{4}$ at a ratio of $\left.1: 3\right)$ or strong base $(\mathrm{NaOH}, \mathrm{pH} 14)$ in a sonication bath (Model 75D, VWR) for 3 hours and then stirred at $90^{\circ} \mathrm{C}$ for 10 hours. The excess acids, bases, or impurities were removed by repeated sonication, centrifuging, and decantion. TEM (Hitachi H-7600), Raman (514.5nm laser, Renishaw, inVia reflex microRaman), and FTIR (Perkin Elmer, Spectrum One) were used to monitor the purification process to characterize the nanodiamonds before and after the purifications. Example spectra for the NDs before and after the acid treatment are given in Fig. S1, which show an increase in the ND purity after the acid purification.

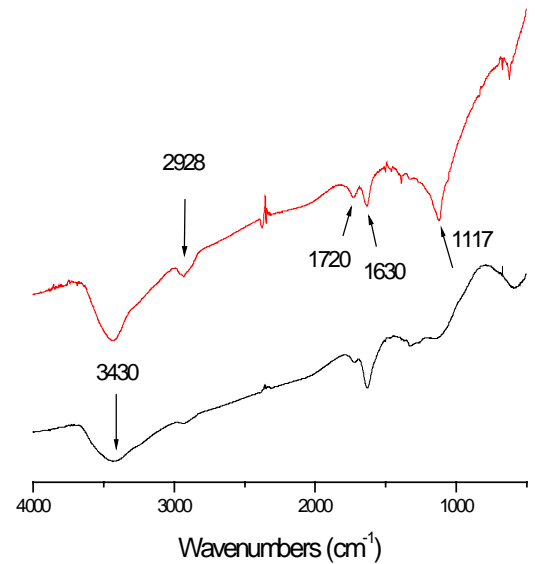

(A)

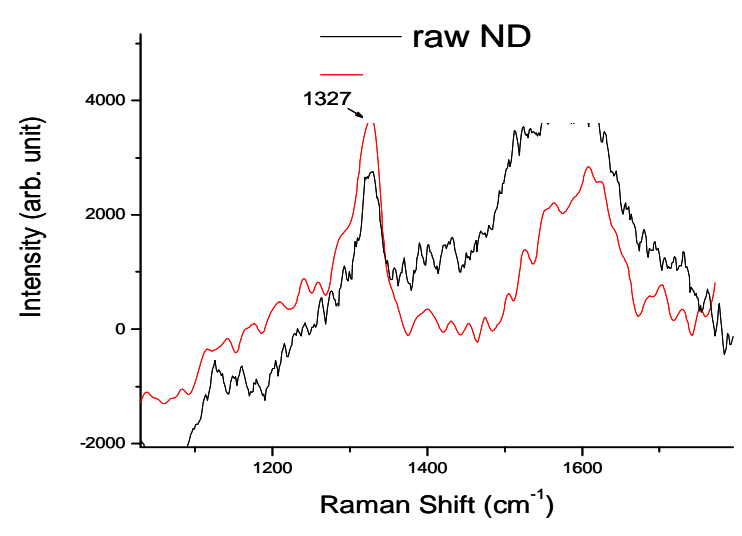

(B)

Figure S1. (A) FTIR and (B) Raman spectra of nandiamonds before and after acid purification. (black: raw; red: purified)

Nanoparticle Preparation and Characterization. All nanoparticles were prepared into stock concentrations of $1 \mathrm{mg} / \mathrm{ml}$ in deionized water (Fig. S2A-D). After dilution with cell culture media into working solutions, ranging in concentration from 5 to 100 
$\mu \mathrm{g} / \mathrm{ml}$ (Fig. S2E-H), the solutions typically increased in turbidity with increasing concentration. However, the acid-functionalized nanodiamonds (ND-COOH, Fig. S2F) were stably suspended and transparent in solution up to $100 \mu \mathrm{g} / \mathrm{ml}$ compared to ND-raw (Fig. S2E), which slightly increased in turbidity at the highest concentration of 100 $\mu \mathrm{g} / \mathrm{ml}$. The increase in turbidity of ND-raw was likely due to agglomerates that may have remained in solution after sonication for dispersion. In contrast, fine carbon black (CB, Fig. S2H) became increasingly dark and turbid with increasing concentration. All nanoparticle concentrations were limited to $100 \mu \mathrm{g} / \mathrm{ml}$ in these studies. Information on nanoparticle dispersion and agglomeration were further examined with light microscopy. Acid-functionalized nanodiamonds (ND-COOH, Fig. S2J) and CdO (Fig. S2L) were found to be well-dispersed whereas ND-raw and CB samples showed aggregrates up to 10's of microns in size (Fig. S2I, K). 
A

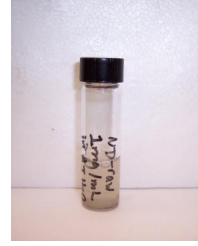

B

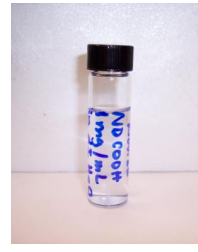

C

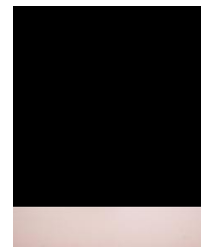

D

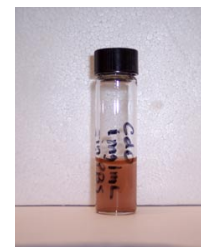

E

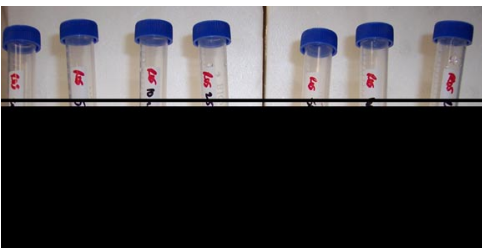

F

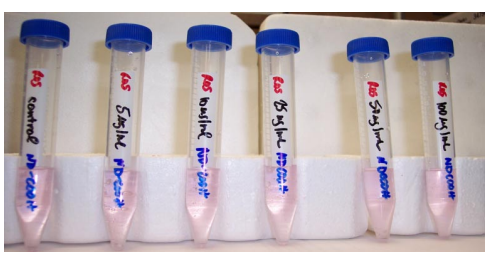

G

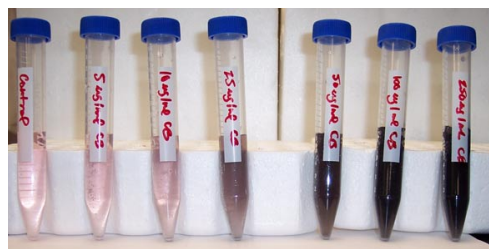

H

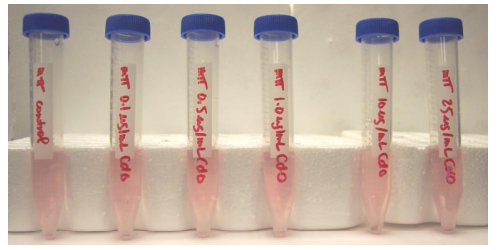

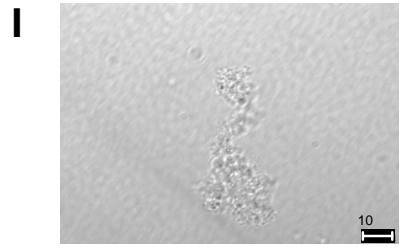

J

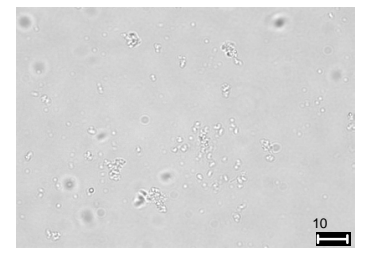

K

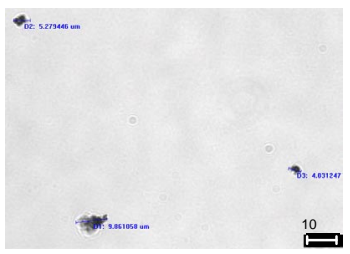

L

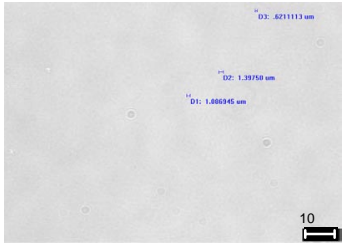

Figure S2. Digital and Light Microscopy Nanomaterials Characterization. (A-D) $1 \mathrm{mg} / \mathrm{ml}$ stock solutions of nanoparticles in aqueous solutions (A) ND-raw in dI water, (B) ND-COOH in dI water, (C) $\mathrm{CdO}$ in saline, and (D) $\mathrm{CB}$ in dI water; (E-H) Nanoparticle dilutions in cell culture media $(\mathrm{E}, \mathrm{F}$, and $\mathrm{H})$ concentrations $0,5,10,25,50,100 \mu \mathrm{g} / \mathrm{ml}$ and $(\mathrm{G})$ concentrations $0,0.1,0.5$, $1,10,25 \mu \mathrm{g} / \mathrm{ml}$; (I-L) Light microscopy examination of dispersion and agglomeration in 25 $\mu \mathrm{g} / \mathrm{ml}$ solutions (I) ND-raw, (J) ND-COOH, (K) CdO, and (L) CB. 

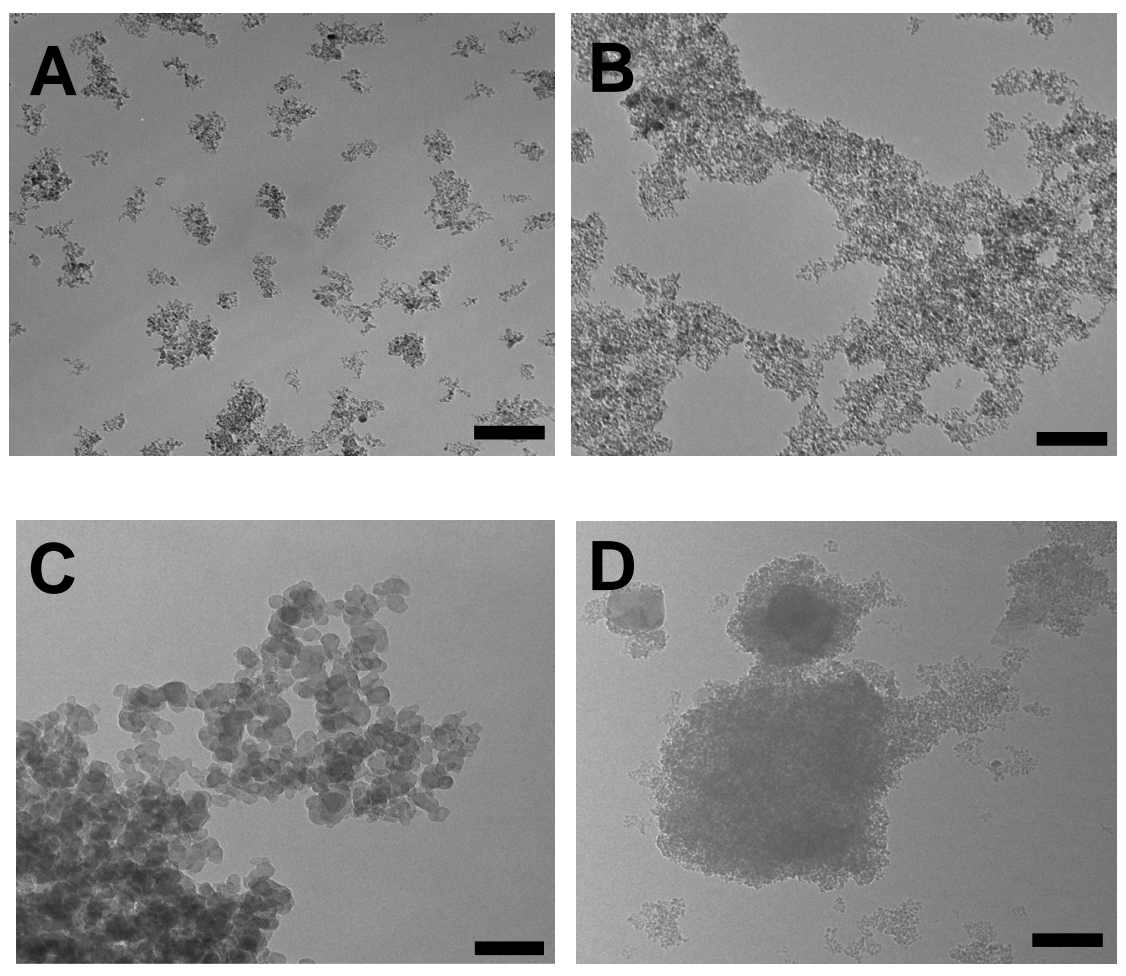

Size Distribution of NDraw

Size Distribution of ND-COOH
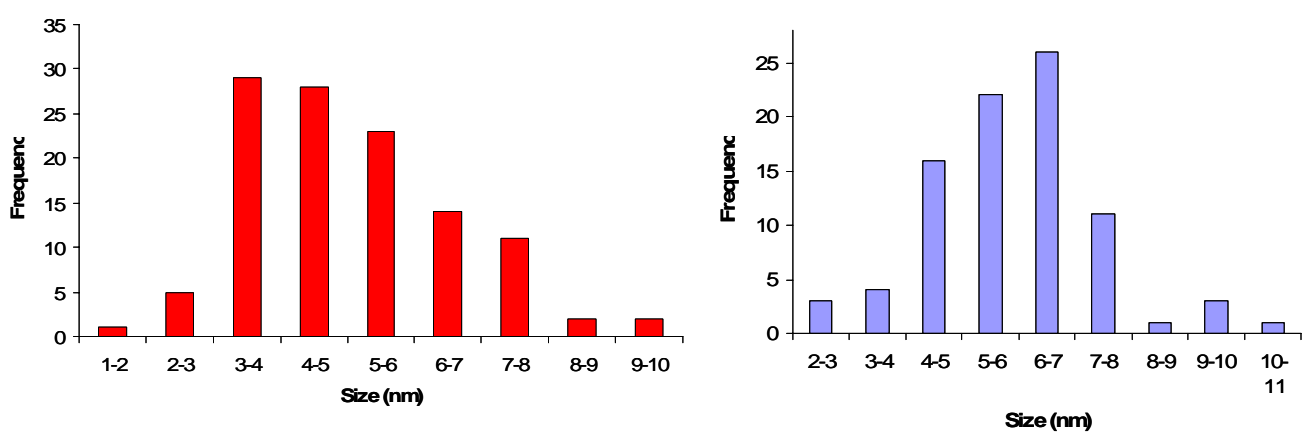

Size Distribution of $\mathrm{CB}$

Size Distribution of CdO
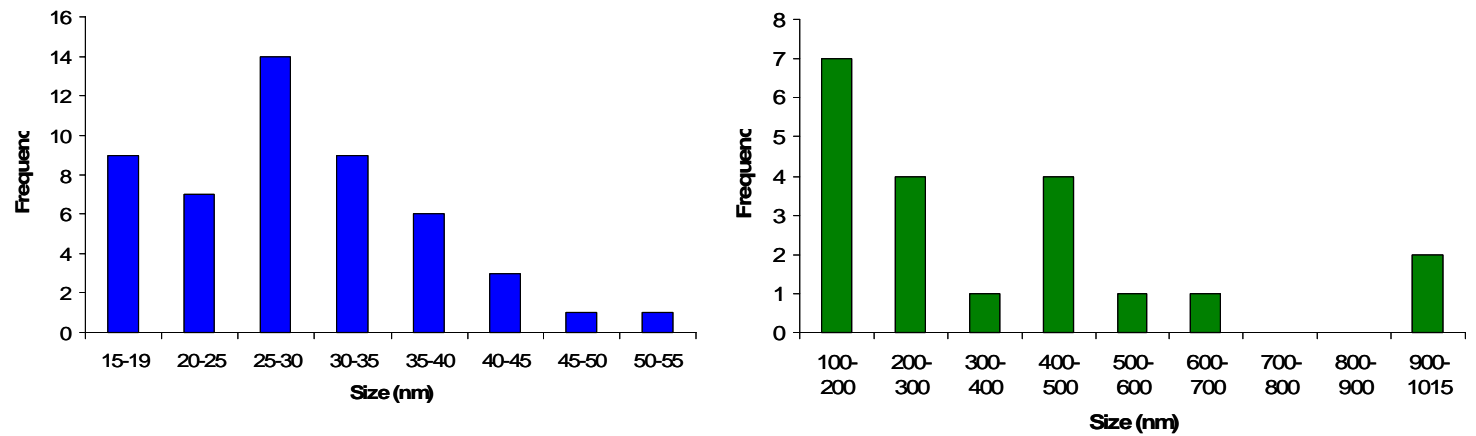

Figure S3. Transmission electron microscopy nanoparticle characterization for morphology and size distribution. (A) ND-raw, (B) ND-COOH, (C) CB, and (D) CdO. 
Higher magnification characterization of nanoparticle size distributions and morphologies was accomplished with transmission electron microscopy (TEM, Fig. S3). Individual nanoparticles or agglomerates were measured from multiple random fields of view. ND-raw nanoparticles were on average $5.1 \pm 1.7 \mathrm{~nm}$ in size (Fig. S3A), ND$\mathrm{COOH}$ nanoparticles were $5.9 \pm 1.6 \mathrm{~nm}$ (Fig. S3B), fine carbon black nanoparticles were $28.8 \pm 8.4 \mathrm{~nm}$ (Fig. S3C), and $\mathrm{CdO}$ nanoparticles were found typically in agglomerates with an average size of $552.1 \pm 238.8$ and core particles of $179.3 \pm 43.4 \mathrm{~nm}$ (Fig. S3D). The formation of very strong ND aggregates, ranging in size from 25 to $100 \mathrm{~nm}$, is a well-known issue ${ }^{1}$. However, the formation of aggregates may merely be an artifact of the drying of nanoparticle solutions before imaging and not representative of true solution dispersion characteristics, which warrants further investigation with techniques such as static or dynamic light scattering.

Nanoparticle Interference Assays. In order to better understand the changes in absorbance on a microplate due to carbon nanoparticles, several experiments were conducted. First, carbon nanoparticles in cell culture media (Fig. S4A), unreduced MTT solution (Fig. S4B), or reduced MTT solution (Fig. S4C) were examined for changes in absorbance on a microplate reader before or after centrifugation to remove the materials. Both ND and CB increase absorbance values when retained in solution. However, the values decreased after they were removed by centrifugation. 


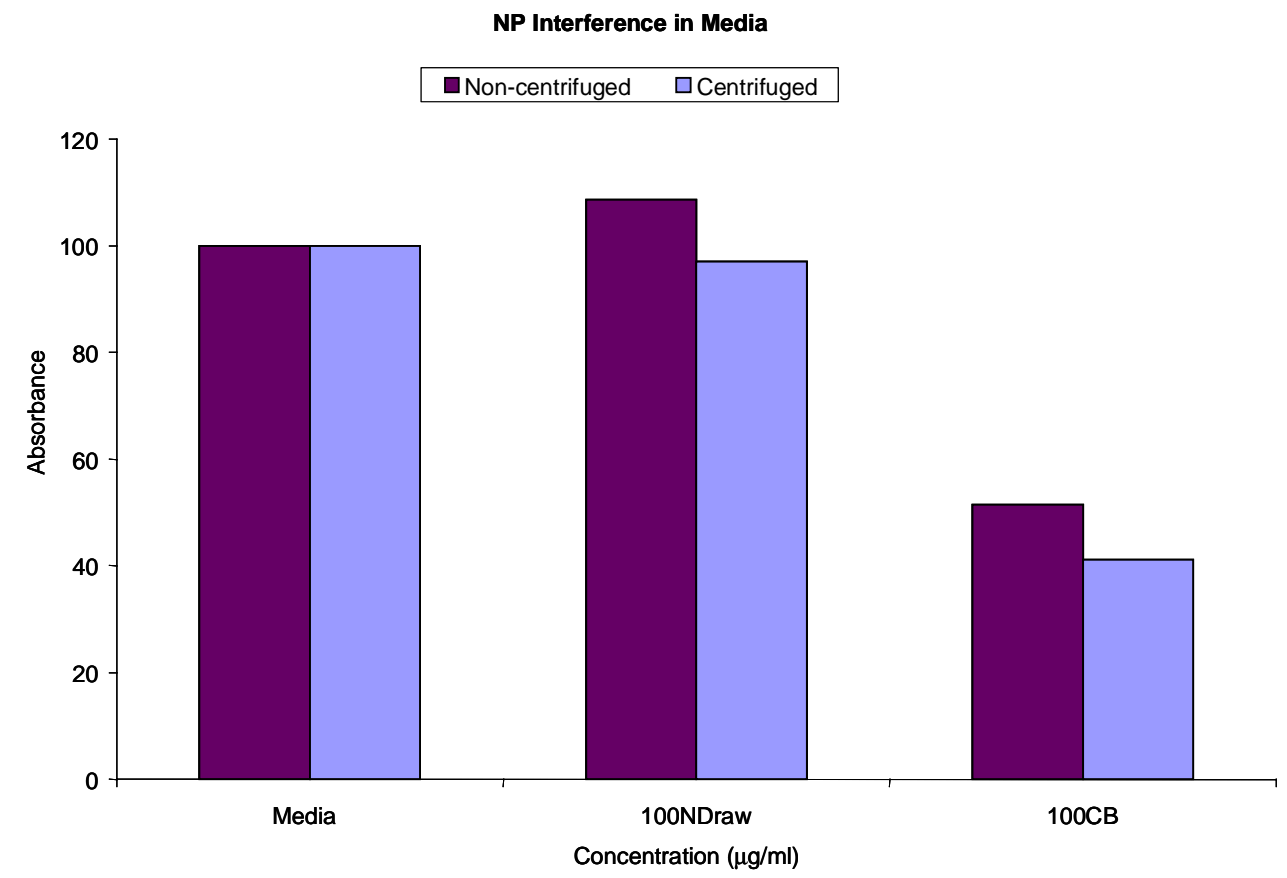

(A)

NP Interference in Unreduced MTT Solution

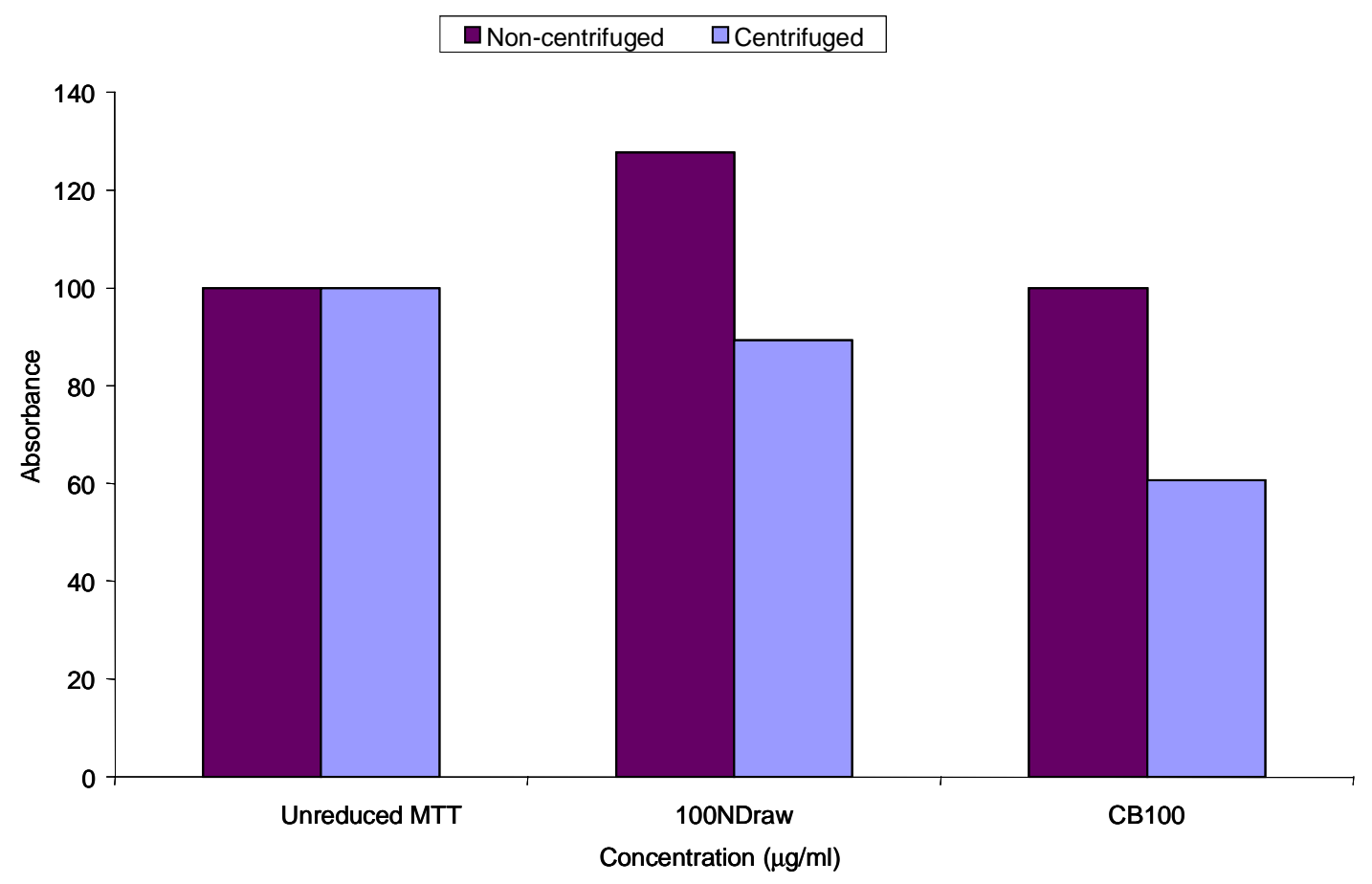

(B) 


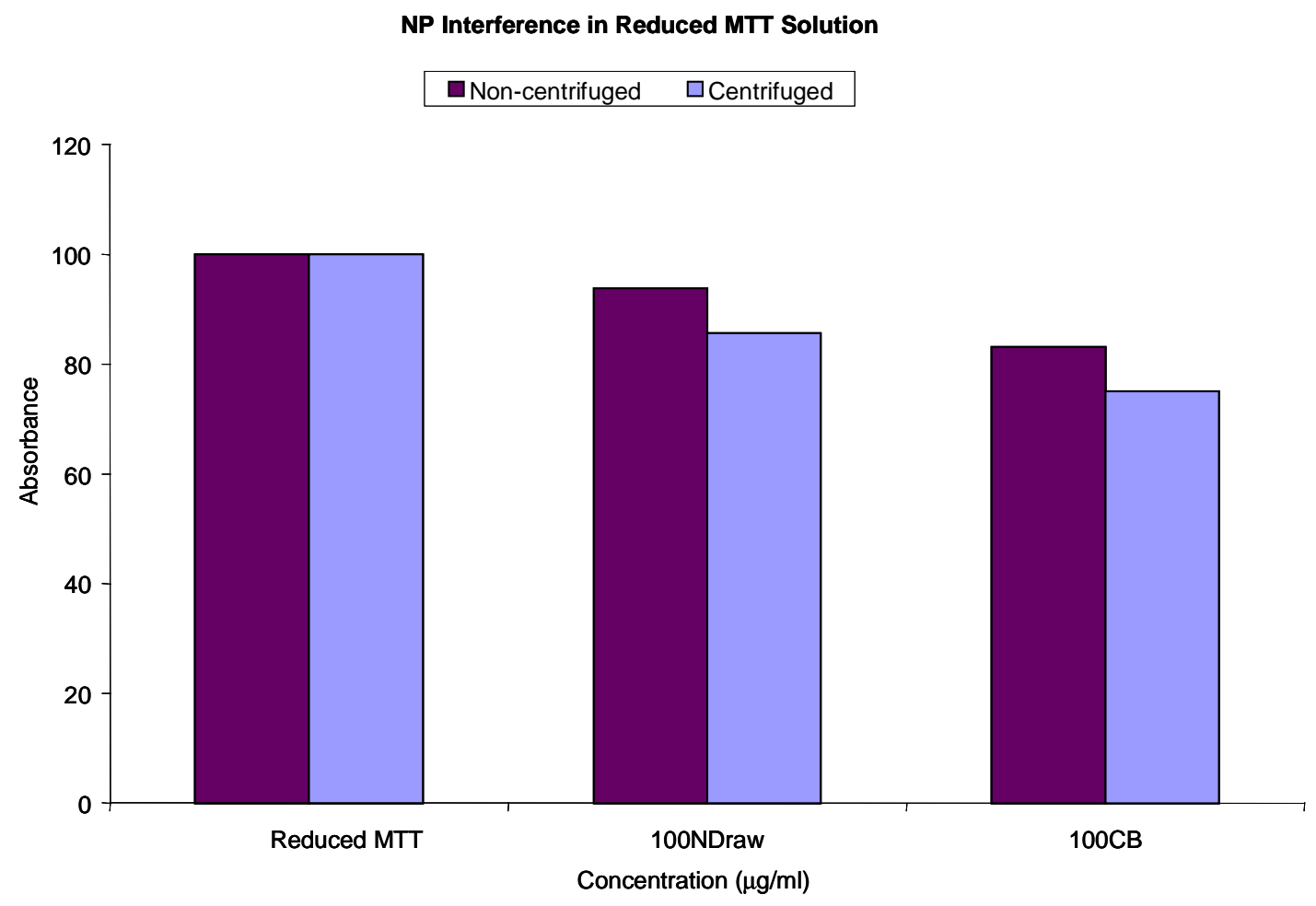

(C)

Figure S4. Effect of Nanoparticles on microplate reader absorbance. (A) nanoparticles in cell culture media, (B) nanoparticles in unreduced MTT solution, and (C) nanoparticles in reduced MTT solution.

Bacterial Growth in ND Waste Solutions. All nanoparticle waste solutions, consisting of nanoparticles, water, and cell culture media, were stored in glass or plastic bottles, which remained at room temperature in non-sterile conditions until $500 \mathrm{ml}$ of waste was accumulated. After 6 months of storage, the only nanoparticle solution to grow a visible amount bacteria was the container with NDs in solution. Neither the carbon nanomaterials (mixture of carbon black, multi-walled, and single-walled carbon nanotubes) nor the $\mathrm{CdO}$ waste solutions contained any appreciable, visible amount of bacterial contamination. An initial observation of the bacteria with light microscopy revealed filamentous and oblong bacteria (Fig. S5 A,B). After staining, the two types of 
bacteria were determined to be gram-positive (Fig. 5 C,D). These observations further support the non-toxic nature of both functionalized and unfunctionalized NDs.
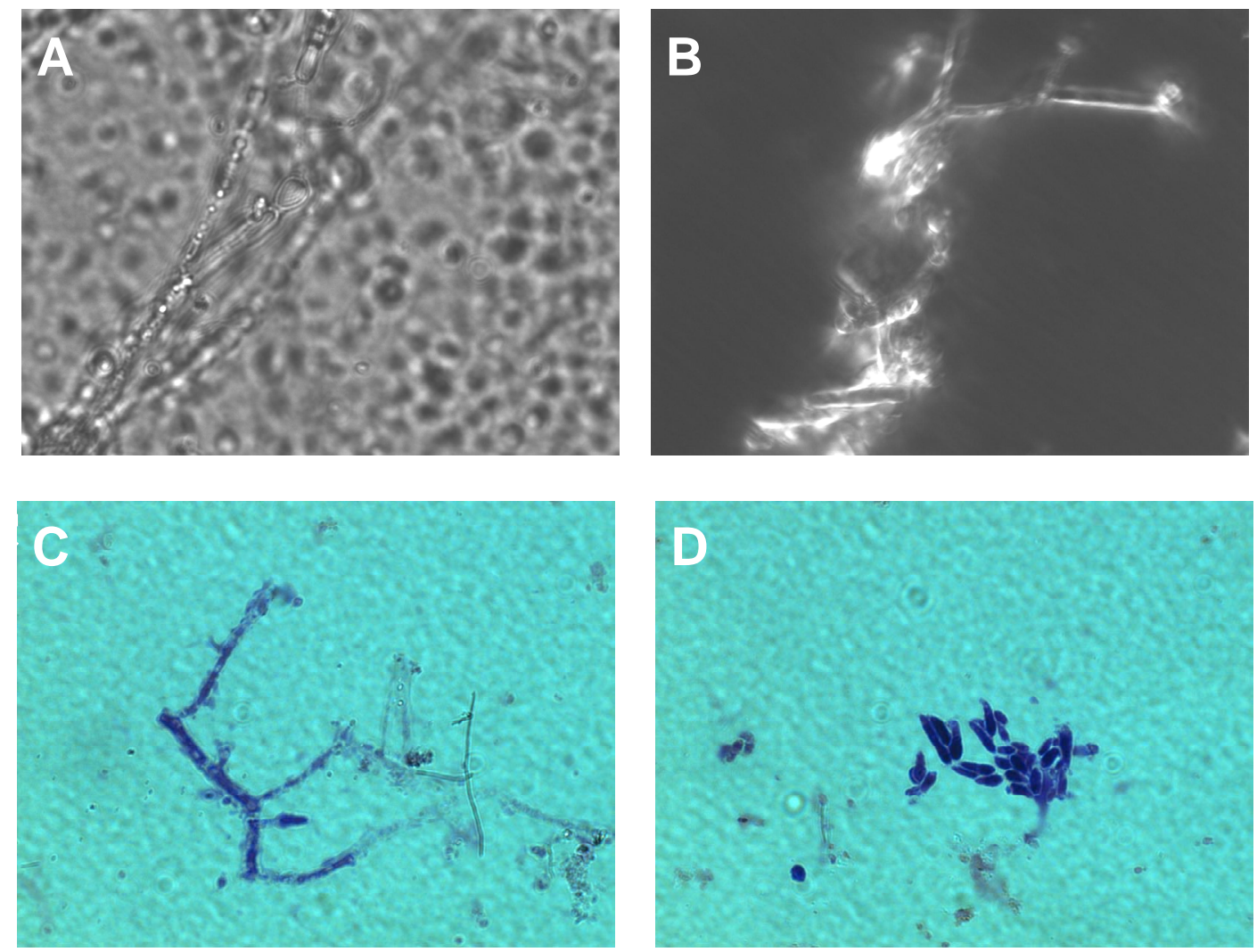

Figure S5. Growth of gram-positive bacteria in waste ND solution imaged with light microscopy at 60x magnifications. (A) Phase contrast image, (B) Cytoviva advanced illumination system attachment for higher resolution image, (C,D) Color images showing gram-positive filamentous and rice-shaped bacteria.

\section{Reference}

Kruger, A.; Kataoka, F.; Ozawa, M.; Fujino, T.; Suzuki, Y.; Aleksenskii, A.; Vul', A.Y.; Osawa, E. Unusually Tight Aggregation in Detonation Nanodiamond: Identification and Disintegration. Carbon 2005, 43, 1722. 